\title{
Altered gut microbiota and inflammatory cytokine responses in patients with Parkinson's disease
}

Chin-Hsien Lin ${ }^{1 *+} \mathbb{D}$, Chieh-Chang Chen ${ }^{2,3+}{ }^{+}$Han-Lin Chiang ${ }^{4}$, Jyh-Ming Liou ${ }^{2}$, Chih-Min Chang ${ }^{5}$, Tzu-Pin Lu ${ }^{6}$, Eric Y. Chuang ${ }^{7,8}$, Yi-Cheng Tai $^{9}$, Chieh Cheng ${ }^{1}$, Han-Yi Lin ${ }^{1}$ and Ming-Shiang Wu ${ }^{2}$

\begin{abstract}
Objective: Emerging evidence suggests that gut microbiome composition alterations affect neurodegeneration through neuroinflammation in the pathogenesis of Parkinson's disease (PD). Here, we evaluate gut microbiota alterations and host cytokine responses in a population of Taiwanese patients with PD.
\end{abstract}

Methods: Fecal microbiota communities from 80 patients with PD and 77 age and gender-matched controls were assessed by sequencing the $\mathrm{V} 3-\mathrm{V} 4$ region of the $16 \mathrm{~S}$ ribosomal RNA gene. Diet and comorbidities were controlled in the analyses. Plasma concentrations of IL-1 $\beta, I L-2, I L-4, I L-6, I L-13, I L-18, G M-C S F$, IFNY, and TNFa were measured by a multiplex immunoassay and relationships between microbiota, clinical characteristics, and cytokine levels were analyzed in the PD group. We further examined the cytokine changes associated with the altered gut microbiota seen in patients with PD in another independent cohort of 120 PD patients and 120 controls.

Results: Microbiota from patients with PD was altered relative to controls and dominated by Verrucomicrobia, Mucispirillum, Porphyromonas, Lactobacillus, and Parabacteroides. In contrast, Prevotella was more abundant in controls. The abundances of Bacteroides were more increased in patients with non-tremor PD subtype than patients with tremor subtype. Bacteroides abundance was correlated with motor symptom severity defined by UPDRS part III motor scores (rho $=0.637$ [95\% confidence interval 0.474 to 0.758 ], $P<0.01$ ). Altered microbiota was correlated with plasma concentrations of IFNY and TNFa. There was a correlation between Bacteroides and plasma level of TNFa (rho $=0.638$ [95\% Cl: 0.102-0.887], $P=0.02$ ); and a correlation between Verrucomicrobia abundance and plasma concentrations of IFNy ( $r h o=0.545[95 \% \mathrm{Cl}-0.043-0.852], P=0.05)$. The elevated plasma cytokine responses were confirmed in an additional independent 120 patients with PD and 120 controls (TNFa: PD vs. control $8.51 \pm 4.63$ $\mathrm{pg} / \mathrm{ml}$ vs. $4.82 \pm 2.23 \mathrm{pg} / \mathrm{ml}, P<0.01$; and IFNY: PD vs. control: $38.45 \pm 7.12 \mathrm{pg} / \mathrm{ml}$ vs. $32.79 \pm 8.03 \mathrm{pg} / \mathrm{ml}, P=0.03$ ).

Conclusions: This study reveals altered gut microbiota in PD and its correlation with clinical phenotypes and severity in our population. The altered plasma cytokine profiles associated with gut microbiome composition alterations suggest aberrant immune responses may contribute to inflammatory processes in PD.

Keywords: Parkinson's disease, Gut microbiome, Dysbiosis, Cytokines, Neuroinflammation

\footnotetext{
* Correspondence: chlin@ntu.edu.tw

${ }^{+}$Chin-Hsien Lin and Chieh-Chang Chen contributed equally to this work.

1 Department of Neurology, National Taiwan University Hospital, College of

Medicine, National Taiwan University, Taipei 100, Taiwan

Full list of author information is available at the end of the article
}

(c) The Author(s). 2019 Open Access This article is distributed under the terms of the Creative Commons Attribution 4.0 International License (http://creativecommons.org/licenses/by/4.0/), which permits unrestricted use, distribution, and reproduction in any medium, provided you give appropriate credit to the original author(s) and the source, provide a link to the Creative Commons license, and indicate if changes were made. The Creative Commons Public Domain Dedication waiver (http://creativecommons.org/publicdomain/zero/1.0/) applies to the data made available in this article, unless otherwise stated. 


\section{Introduction}

Parkinson's disease (PD) is a common neurodegenerative disorder and is caused by a combination of genetic and environmental risk factors [1]. Aggregations of intraneuronal $\alpha$-synuclein known as Lewy Bodies (LB) are pathological hallmarks of this disease. The enteric nervous system (ENS) is among the structures earliest affected by LB pathology in the disease process of PD [2]. Accordantly, gastrointestinal dysfunction, particularly constipation, is the most common non-motor PD symptom and often precedes the motor disability onset by decades [3].

Emerging evidence suggests that gut microbiota may act as environmental triggers to promote neuronal degeneration and motor dysfunction through microglial activation in $\alpha$-synuclein-overexpressing mice [4]. These observations are consistent with the altered gut microbiomes reported in patients with PD [5-12], although the reported gut bacterial profiles are heterogeneous. These inconsistent results may be related to the variable geographic origins and ethnic backgrounds of PD patients included in previous studies. An epidemiology study has shown that the PD incidence and prevalence is lower in Asians than those in Western populations [13], and there is clear evidence for distinct genetic causes of PD between these two populations. For example, the frequency of the prevalent p.G2019S mutation in the leucine-rich repeat kinase 2 (LRRK2) gene accounts for up to $40 \%$ of PD cases in North African Arab-Berbers and Ashkenazi Jews but is found in less than $0.1 \%$ of Asian PD patients [14], suggesting that Asian patients with PD may have different gut microbiota than Western PD patients. Gut microbiome composition alterations are also linked to aberrant immune responses, which are often accompanied by abnormal production of inflammatory cytokines [15], reinforcing the role of neuroinflammation in PD. However, few studies have explored the gut microbiome and related host cytokine responses in patients with PD. Here, we characterize fecal microbiota and peripheral cytokine alterations in a Taiwanese PD population and analyze the relationships between changes of fecal microbiota and PD clinical characteristics.

\section{Methods}

\section{Study participants}

The study flow chart was depicted in Fig. 1. A total of 397 study participants were included in this study, comprising 80 PD patients and 77 age and sex-matched control subjects without evidence of PD in the first part of the study to compare the gut microbiome changes, and an additional independent cohort of 120 patients with PD and 120 age and sex-matched controls were enrolled in the second part of the study to examine the cytokine changes associated with the altered gut microbiota seen in patients with PD. All participants provided informed

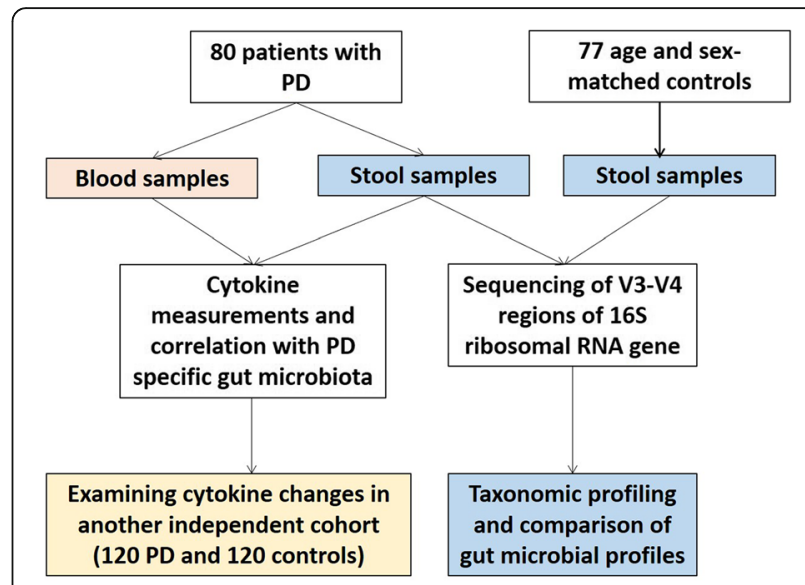

Fig. 1 Flow chart and enrolled participants in the current study

consent and the study was approved by the institutional ethics board of National Taiwan University Hospital.

PD was diagnosed according to the United Kingdom PD Society Brain Bank clinical diagnostic criteria [16]. The motor phenotypes, i.e., tremor or non-tremor predominant type, were practically determined by the initially presenting symptoms of the patients, which was recorded in the medical records. Healthy control subjects, who were neurologically normal patients visiting our clinics, spouses, or accompanied friends of the PD patients, were recruited from the same institute. Participants with the previous diagnosis of inflammatory bowel disorders, irritable bowel syndrome, colitis, or colon cancer were excluded. Individuals currently taking antibiotics or probiotic supplements within 3 months of sample collection were also excluded. A comprehensive dietary history (Food Frequency Questionnaire) with 64 items [17] and medical comorbidities were collected.

\section{Evaluation of motor and non-motor symptoms}

Motor symptom severity was evaluated using the motor sub-scores of the Unified Parkinson's Disease Rating Scale (UPDRS part III) and Hoehn-Yahr staging, which were performed for each subject in both the practically defined "off" medication state (patients willing and able to withhold all PD medications for at least $12 \mathrm{~h}$ prior to assessment) and the "on" medication state ( $1 \mathrm{~h}$ after taking their usual morning PD medications that same day). Nonmotor symptoms were assessed by the Non-Motor Symptom assessment Scale (NMSS), and cognition was examined with the Mini-Mental State Examination (MMSE) during the "on" phase of the PD patients. Constipation was diagnosed using the Rome III Criteria [18]. Furthermore, the severity of constipation was also assessed by a sub-score of the NMSS, which is defined as a frequency of defecation less than three times a week. 


\section{Sample collection and DNA extraction}

Stool samples were collected into stool specimen collection tubes containing DNA stabilizer (Sarstedt), which were immediately flash-frozen on dry ice, and stored at $-80^{\circ} \mathrm{C}$ until analysis. Total fecal DNA was extracted using a QIAamp DNA Stool Mini Kit (Qiagen, Hilden, Germany) as previously described [8]. Blood samples of $10 \mathrm{ml}$ of venous blood were drawn from each participant at enrolment and were centrifuged ( $2500 \mathrm{~g}$ for $15 \mathrm{~min}$ ) within $1 \mathrm{~h}$ of collection. The plasma was aliquoted into cryotubes following centrifugation and stored at $-80^{\circ} \mathrm{C}$ for cytokine analysis.

\section{$16 \mathrm{~S}$ ribosomal RNA gene amplicon and sequencing}

We used universal primers linked with indices and sequencing adaptors to amplify the V3-V4 regions of the bacterial $16 \mathrm{~S}$ ribosomal RNA (16S rRNA) gene after extraction of total DNA from the fecal samples. The amplicons were sequenced on an Illumina Miseq platform to obtain 300-bp paired-end reads and for taxonomic assignment. Detailed descriptions of the amplicons and the sequencing analysis protocol are provided in the Additional file 1: Supplementary Methods.

\section{Measurement of plasma cytokine levels by a multiplex immunoassay}

The ProcartaPlex Human Th1/Th2 Cytokine Panel 11plex (Thermo Fisher Scientific, Vienna, Austria) was used to measure plasma concentrations of IL-1 $\beta$, IL-2, IL-4, IL-6, IL-13, IL-18, GM-CSF, IFN $\gamma$, and TNF $\alpha$ according to the manufacturer's instructions.

\section{Statistical analyses}

Comparisons between groups were performed with Student's $t$ tests and chi-square tests for quantitative and categorical variables, respectively. Variables that followed a Gaussian distribution were compared with two-tailed $t$ tests or analysis of variance (ANOVA). We tested the homogeneity of variances by using Levene's test. For variables that violated the assumptions of normality or homoscedasticity, the groups were compared with nonparametric Mann-Whitney $U$ test (for two groups) or Kruskal-Wallis test (for more than two groups).

Differences were tested at the operational taxonomic units (OTUs), genus, and family levels. Taxa present in < $10 \%$ of samples were removed. The relative abundance of each taxon in PD patients versus controls, the Chaol diversity index, and the Shannon entropy index were examined using the analysis of composition of microbiomes (ANCOM) and Kruskal-Wallis rank sum test, which both tests were conducted using default parameters in the python implementation of ANCOM in scikit-bio 0.4.2 (http://scikit-bio.org/docs/0.4.2/index.html) and the Kruskal-Wallis test was run using kruskal.test in $\mathrm{R}$ package. Correlations between plasma cytokine concentrations and genera expressed sequence counts with a prevalence $>10 \%$ were calculated using Spearman's rank-correlation analysis under the assumptions that there is a non-linear relationship between the examined variables. The genera were then subjected to generalized linear model (GLM) analysis with a negative binomial distribution and controlling for zero-inflation as appropriate in the $\mathrm{R}$ package glmmADMB. Several factors were adjusted for different confounders including age, sex, and diet (the mean daily intake amount of protein, carbohydrates, total fat, and dietary fiber). Correlations between PD clinical parameters or plasma cytokine concentrations and genera expressed sequence counts with a prevalence $>10 \%$ were calculated using Spearman's rank-correlation analysis for $80 \mathrm{PD}$ patients under the assumptions that there is a non-linear relationship between the examined variables. All analyses were performed with Stata (StataCorp LP, College Station, USA) software and R software (version 3.1.0, the R Project for Statistical Computing). A $P$ value $<0.05$ was considered significant.

\section{Results \\ Clinical characteristics of PD and control groups}

The demographic and clinical information of 80 patients with PD and 77 control subjects enrolled in the first step of the study are summarized in Additional file 2: Table S1. A higher proportion of the PD group reported constipation than the controls.

(66.3\% vs $12.3 \%, P<0.01)$. The mean UPDRS part III scores and Hoehn-Yahr stages during the "on" and "off" state as well as NMSS scores are also included for patients with PD. There was no difference in age or sex between the two groups. There were more medical comorbidities including diabetes mellitus and hypertension in controls than in patients with PD. Of the clinical variables, the intensity of constipation was higher in PD patients with more advanced Hoehn-Yahr stages than in PD patients at early motor stages $(P<0.001$ by one-way ANOVA).

\section{Patients with PD have altered and more diverse gut microbiota}

We then characterized the bacterial gut microbiota associated with PD by high-throughput sequencing of the V3$\mathrm{V} 4$ region of the $16 \mathrm{~S}$ ribosomal RNA gene. We measured the bacterial richness within each sample from both the PD and control groups using three different methods, the observed number of operational taxonomic units (OTUs), the Chaol diversity index, and the Shannon entropy index. The bacterial gut microbiota from patients with PD was more diverse than those from controls by all the three estimators $(P=0.08$ for the Chaol diversity index, $P=0.02$ for the observed species diversity index, and $P<0.001$ for the Shannon entropy index; by Wilcoxon rank-sum test, Fig. 2a). 

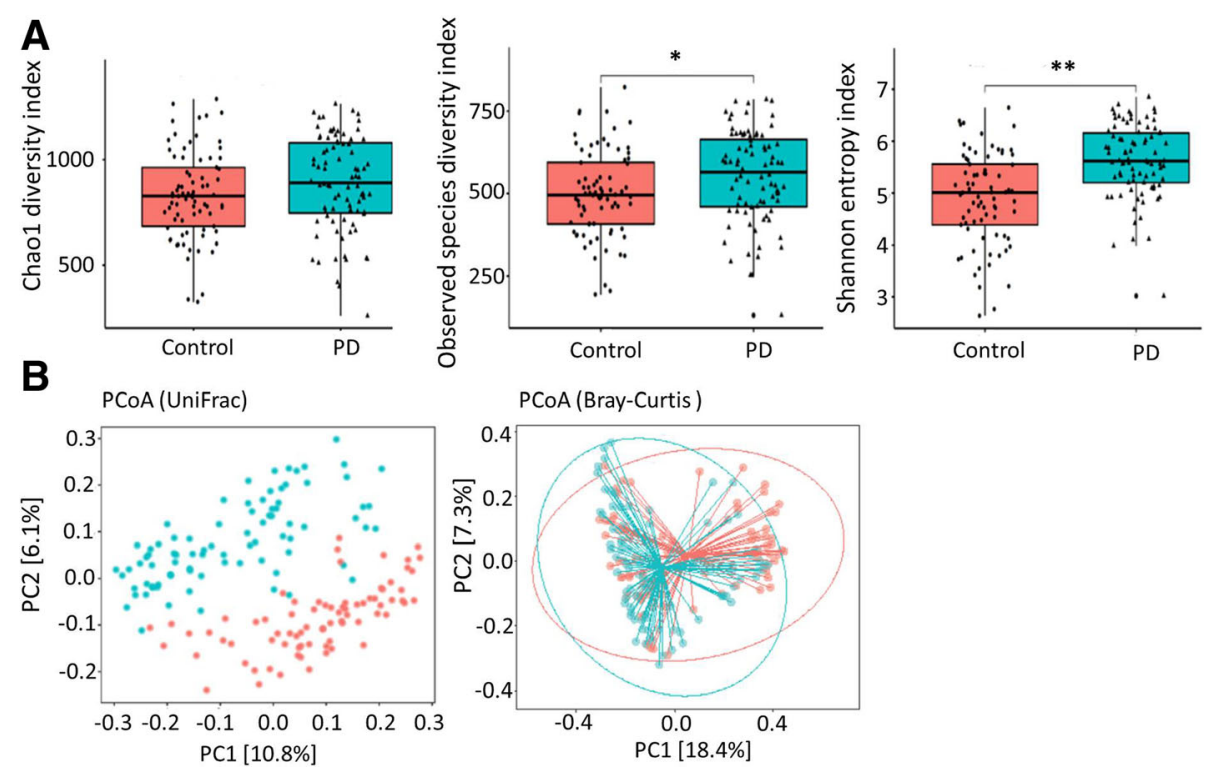

Fig. 2 The a-diversity and $\beta$-diversity indices of the fecal microbiome in the PD and control groups. a Box plots depict differences in the fecal microbiome diversity indices between the PD and control groups according to the Chao 1 index, observed species index, and Shannon index based on OTU counts. Each box plot represents the median, interquartile range, minimum, and maximum values. OUT: operational taxonomic units (b) PCoA plots of bacterial $\beta$-diversity based on the weighted UniFrac distance (left panel) and Bray-Curtis dissimilarity (right panel) analyzed according to health status. Patients with PD and age-matched controls are colored in blue and red, respectively

We calculated the $\beta$-diversity of the samples using the weighted UniFrac distances and the Bray-Curtis dissimilarity to identify possible differences between the bacterial components in the gut microbiota of patients with $\mathrm{PD}$ and controls. The principal coordinates analysis $(\mathrm{PCoA})$ revealed that the gut microbiota of patients with PD was distinct from those of the controls $(P<0.001$ by a Permutational MANOVA (PERMANOVA) implementation using Uni-Frac distances and Bray-Curtis dissimilarity, Fig. 2b). These findings indicate that the richness and diversity of the gut microbiota in patients with PD are significantly different from that of controls.

\section{Alteration in gut microbiota between PD and control groups}

A supervised comparison of the microbiota between PD and control groups was performed by linear discriminant analysis (LDA) effect size (LEfSe) analysis without any adjustments, which is often used to identify the presence and effect size of region-specific OTUs among different groups (Additional file 1: Supplementary Methods) [19]. We used a logarithmic LDA score cutoff of 2.0 to identify important taxonomic differences between the PD and control groups and found a notable difference in fecal microbiota between the PD and control groups based on LDA LEfSe analysis (Fig. 3a). We observed that the relative abundance of the Prevotella genus was higher in the control group than in the PD group, while the relative abundances of Parabacteroides,
Verrucomicrobia, Akkermansia, Butyricimonas, Veillonella, Odoribacter, Mucispirillum, Bilophila, Enterococcus, and Lactobacillus were higher in patients with PD than in controls (LDA score $(\log 10)>2$, Fig. 3a, b) . GLMs with negative binomial distribution for bacterial abundances defined by sequence counts were used to model genera that were significantly different between the two groups after controlling for possible confounding factors such as age, sex, and diet (the mean daily intake amount of protein, carbohydrates, total fat, and dietary fiber). Microbiome differences between the groups were associated with Verrucomicrobia, Prevotella, Mucispirillum, Porphyromonas, Lactobacillus, and Parabacteroides $(P<0.05$, Additional file 2: Table S2), suggesting an association between these genera and PD. The mean abundance of Prevotella was reduced by $46.6 \%$ in patients with PD compared to controls (Fig. 3c). The other genera were more abundant in patients with PD than in controls, but the absolute differences between the groups were smaller than that observed for Prevotella (data not shown).

\section{Association between fecal microbiota and PD clinical characteristics}

We next examined the association between fecal microbiota and PD clinical subtypes. Of 80 PD patients, 47 $(58.8 \%)$ were initially presented with tremor while the others presented with non-tremor subtypes, including the akinetic-rigidity or postural instability and gait difficulty (PIGD) subtypes. We found a significant difference 


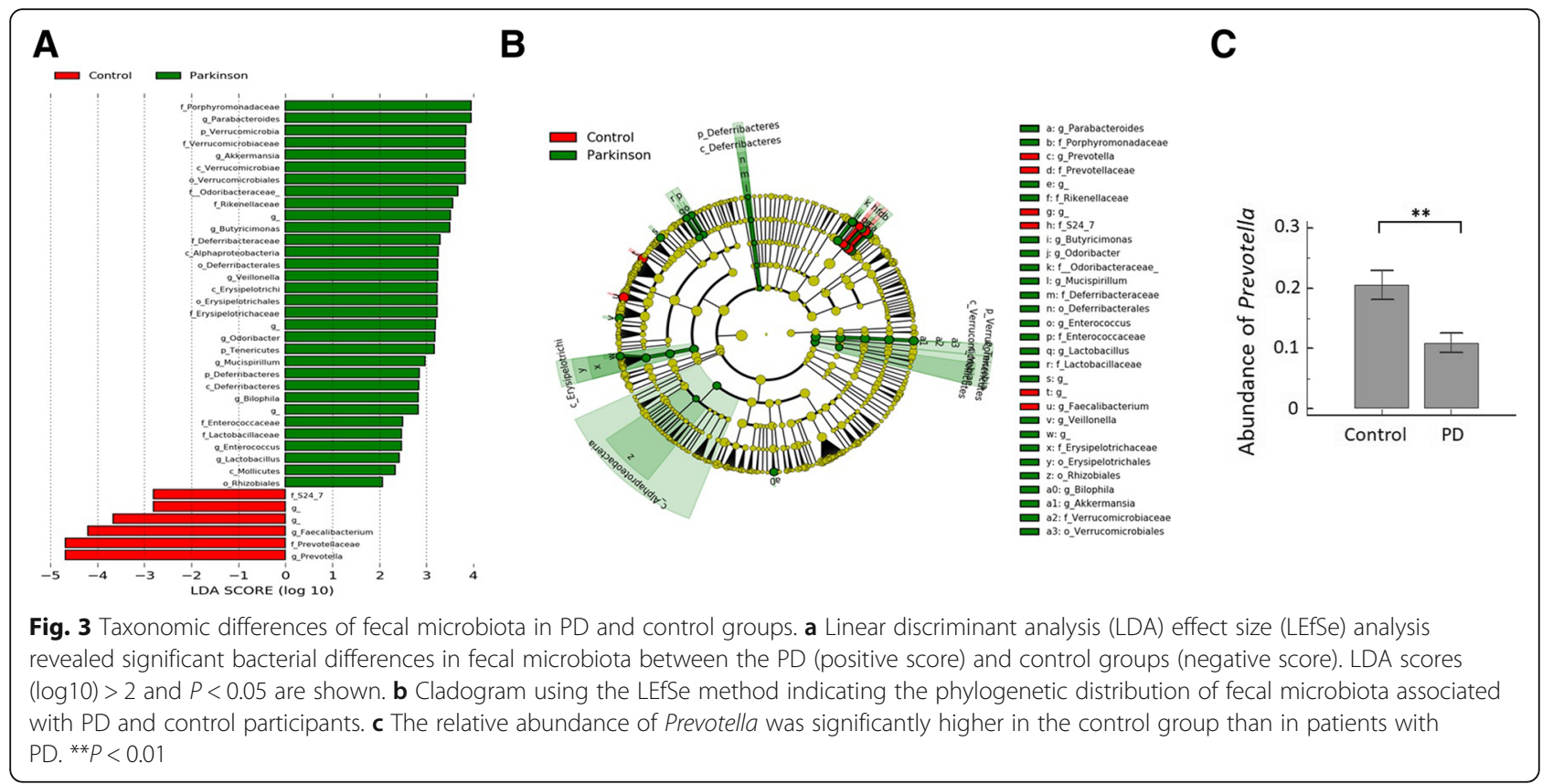

in fecal microbiota between the tremor and non-tremor subtypes based on LDA LEfSe analysis without specific confounder adjustment (Fig. 4a). GLMs with negative binomial distribution for bacterial abundances defined by sequence counts were used to model genera that were significantly different between tremor and non-tremor subtypes after controlling for possible confounding factors such as age, sex, and diet (the mean daily intake amount of protein, carbohydrates, total fat, and dietary fiber). The relative abundances of Clostridium, Verrucomicrobia, and
Akkermansia were higher in the tremor subtype than in the non-tremor subtypes, whereas the relative abundances of Propionibacterium, Bacteroidia, Flavobacterium, Mogibacterium, Sutterella, Alcaligenacea Cupriavidus, and Desulfovibrio were higher in the non-tremor subtype (LDA score $(\log 10)>2$, Fig. 4a, b). Of these classes and genera, the mean abundance of Bacteroides genus from Bacteroidia class was increased by $41.6 \%$ in patients with non-tremor subtype PD compared to patients with the tremor-subtype (Fig. 4c). Bacteroides abundance also

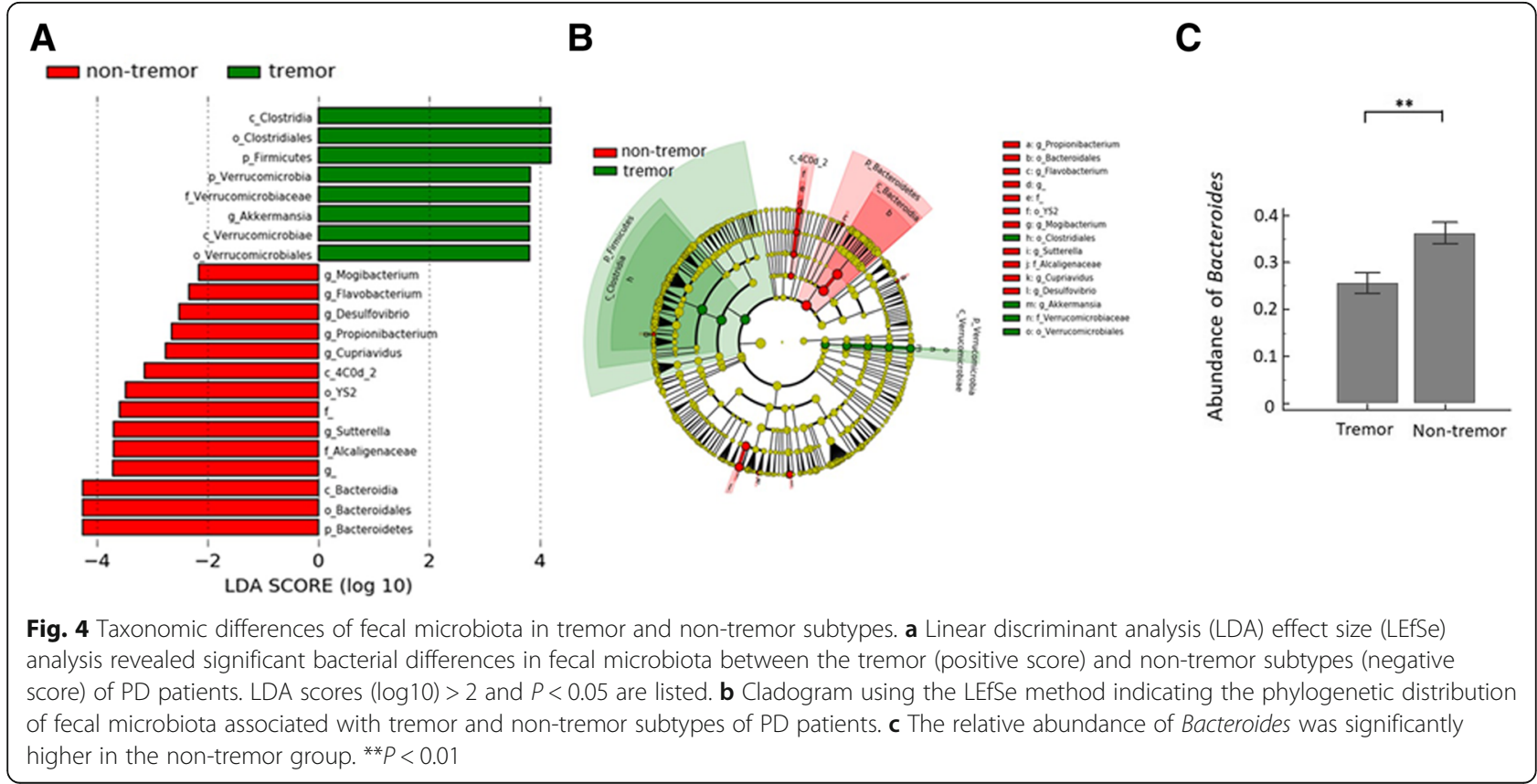


correlated with motor symptom severity as defined by UPDRS part III motor scores (rho $=0.637$ [95\% confidence interval 0.474 to 0.758 ], $P<0.01$ by Spearman correlation analysis).

\section{Altered cytokine responses in PD patients with alterations in taxonomic compositions of the gut microbiota}

Because gut microbial dysbioses are often accompanied by abnormal production of inflammatory cytokines such as IL-1 $\beta$, IL-22, and IFN $\gamma[15,20]$, we next investigated whether there are specific cytokine responses correlated with the relative abundances of the candidate genera associated with risk for or motor severity of PD, including Verrucomicrobia, Prevotella, Mucispirillum, Porphyromonas, Lactobacillus, Parabacteroides, and Bacteroides. Among the nine cytokines we examined in the human Th1/Th2 cytokine panel, we found there was a correlation between Bacteroides and plasma concentrations of TNF $\alpha \quad$ (rho $=0.638 \quad[95 \%$ CI $0.102-0.887], \quad P=0.02$, Fig. 5a); and a correlation between Verrucomicrobia abundance and plasma concentrations of IFN $\gamma$ (rho = 0.545 [95\% CI - 0.043-0.852], $P=0.05$, Fig. 5b). Consistently, the plasma levels of TNFa and IFN $\gamma$ were increased in the PD group than controls (TNFo: PD vs. control: $10.71 \pm 3.89 \mathrm{pg} / \mathrm{ml}$ vs. $6.04 \pm 2.73 \mathrm{pg} / \mathrm{ml}, P=$ 0.02; and IFNY: PD vs. control: $37.22 \pm 9.15 \mathrm{pg} / \mathrm{ml}$ vs. $28.39 \pm 10.21 \mathrm{pg} / \mathrm{ml}, P<0.01$ ) (Fig. 5c).

We then evaluated plasma cytokine responses in an additional independent 240 participants, including 120 patients with PD $(62.3 \pm 7.8$ years, $62.5 \%$ men $)$ and 120 age and sex-matched controls $(61.8 \pm 8.3$ years, $60.0 \%$ men) to confirm the altered cytokine responses in the PD disease state. The plasma concentrations of TNF $\alpha$ and IFN $\gamma$ were consistently and significantly higher in patients with PD than in controls (TNFa: PD vs. control: $8.51 \pm 4.63 \mathrm{pg} / \mathrm{ml}$ vs. $4.82 \pm 2.23 \mathrm{pg} / \mathrm{ml}, P<0.01$; and
IFNY: PD vs. control: $38.45 \pm 7.12 \mathrm{pg} / \mathrm{ml}$ vs. $32.79 \pm 8.03$ $\mathrm{pg} / \mathrm{ml}, P=0.03$; both were analyzed by one-way ANOVA) (Fig. 5). Additionally, the plasma level of IL-13 showed a marked increment in PD compared to the control group $(12.49 \pm 6.55 \mathrm{pg} / \mathrm{ml}$ vs. $2.93 \pm 0.52 \mathrm{pg} / \mathrm{ml}, P<0.01$; by oneway ANOVA, Additional file 3 ). These results suggest that the altered microbiota seen in patients with PD may be associated with systemic inflammatory responses that contribute to PD development (Additional file 3).

\section{Discussion}

Our study identified a clear gut microbiome composition alteration in individuals affected by PD in a large sample size recruited from an Asian population. The relative abundances of Verrucomicrobia and Bacteroides, which was either increased in PD patients or associated with motor symptom severity, correlated with elevated plasma concentrations of TNF $\alpha$ and IFN $\gamma$ in patients with PD suggesting the occurrence of a systemic subinflammatory status associated with altered gut microbiota. Our results not only demonstrate that specific genera of gut microbiota may associate with the risk and severity of PD in our population, but also extend the current knowledge to glimpse the complexity of the interaction between gut microbiome composition alterations and host immune responses in PD pathogenesis.

We show that the richness and diversity of fecal microbiota as defined by $\alpha$-diversity indexes were altered in patients with PD compared to those in the control group, consistent with previous reports from the USA [9] as well as another independent Chinese population [21]. The microbiome structures defined by the $\beta$ diversity index were qualitatively different between the PD and control groups. These observations were consistent with previous evidence suggesting that gut microbiota is altered in patients with PD [5, 8-10, 21, 22].
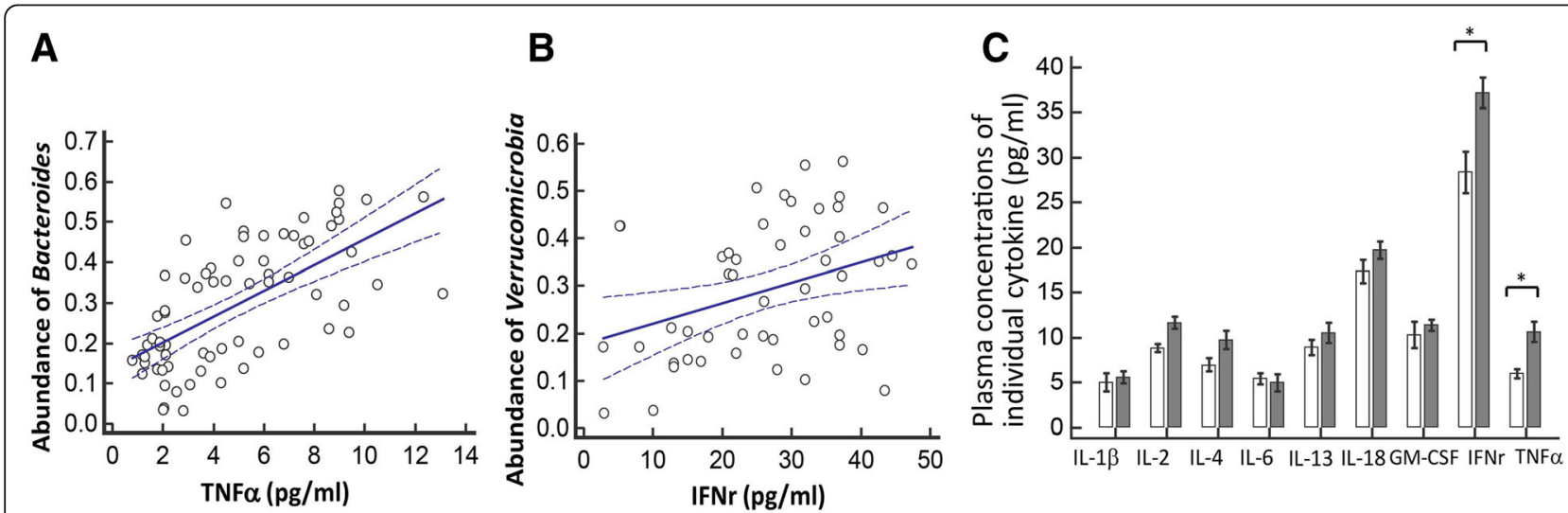

Fig. 5 Correlations between abundances of specific fecal microbiota and plasma levels of cytokines. a The correlation between the relative abundances of Bacterioides and plasma concentration of TNFa. $\mathbf{b}$ The correlation between the relative abundances of Verrucomicrobia and plasma level of IFNy. c Plasma concentrations of individual cytokines in PD and control groups in the firsts set of the study. The error bar indicated standard error of mean (SEM). PD, Parkinson's disease. ${ }^{*} P<0.05 .{ }^{* *} P<0.01$ 
Although diets and ethnic backgrounds were distinct between Asian and Western populations, we identified some common genera changing in abundance in PD patients relative to controls. Our study observed that Prevotella abundance was reduced in patients with $\mathrm{PD}$, in agreement with previous studies conducted in Germany, Finland, Russia, and Japan [5, 8, 10, 23, 24]. Trends toward a decrease in Prevotellacea were also reported in one study investigating the microbiome of the colonic mucosa rather than feces in PD patients [9]. Prevotella is a commensal microbe in the colon that maintains mucin glycoproteins in the gut mucosal layer but may also interact with the immune system [25]. Although some emerging studies in humans have linked the increased abundance of Prevotella in other systemic diseases, including periodontitis, bacterial vaginosis, rheumatoid arthritis, metabolic disorders, and low-grade systemic inflammation, a decreased Prevotella abundance is consistently observed in PD patients [5, 8-10, 23, 24]. The decreased Prevotella abundance is consistent with previous observations of increased gut permeability in PD, since low Prevotella levels may indicate decreased mucin synthesis, which is associated with leaky gut and aberrant gut immune responses and can lead to neurodegeneration in PD [7]. Decreased Prevotella has also been linked to reduced ghrelin concentration, and altered ghrelin secretion was reported previously in one PD study [5]. Together, our results suggest a possible protective role for Prevotella against neurodegenerative processes in PD. However, due to the conflicting role of Prevotella in other systemic medical disorders, the role of this genus in PD pathogenesis should be investigated further.

The relative abundance of the Bacteroides genus was higher in patients with the non-tremor PD subtype and correlated with motor symptom severity as defined by UPDRS part III motor sub-scores. Since PD is a clinically heterogeneous disorder, patients with the nontremor subtype progress faster and show more severe $\alpha$ synuclein pathology in colonic ENS neurons than patients with tremor [26]. The Bacteroidetes constitutes the largest phylum of Gram-negative bacteria in the gastrointestinal tract microbiome and some genera of Bacteroidetes, such as Bacteroides fragilis, has the potential to secrete a remarkably complex array of proinflammatory neurotoxins including surface lipopolysaccharides (LPSs) and toxic proteolytic peptides [27]. One recent microbiota analysis in fecal and colonic mucosa samples from patients with multiple system atrophy, another neurodegenerative disorder involving the nigrastriatal system, also revealed a relatively high abundance of Bacteroidetes [28]. We further identified a positive correlation between the abundance of Bacteroides genus from Bacteroidetes phylum and the plasma concentration of the pro-inflammatory cytokine TNF $\alpha$ in our PD patients. Bacteroides species have been shown to stimulate macrophages and monocytes to secrete TNF $\alpha$ by LPSmediated pathways [29]. Consistently, one recent study identified elevated stool inflammatory profiles, such as interleukin-1 and CXCL8, in patients with PD compared to controls [30]. Future studies are needed to uncover the interactions between commensal gut microbiota changes and the immune reactions in PD pathogenesis.

We also found increased relative abundances of Verrucomicrobia, Mucispirillum, Porphyromonas, Lactobacillus, and Parabacteroides in the fecal microbiomes of patients with PD than in those of controls after adjusting for age and gender, consistent with previous reports of increased Verrucomicrobia and Lactobacillus abundance in patients with PD with diverse ethnicities $[8,11,21,22]$. We also identified a modest correlation between Verrucomicrobia abundance and plasma concentrations of IFNy, a proinflammatory cytokine produced by type I helper $\mathrm{T}$ cells [31]. Because altered gut microbiota are linked to aberrant gut and systemic immune responses, often accompanied by the abnormal production of inflammatory cytokines in the blood, our observations support a previous study showing that gut microbiota promoted motor deficits and neurodegeneration by activating neuroinflammation in an $\alpha$ synuclein-overexpressing PD mouse model [4]. Additional functional studies are needed to elucidate the relationship between altered gut microbiome compositions and the immune reaction in the pathogenesis and progression of PD.

The major advantage of this study is the relatively large sample size from an East Asian population. Additionally, we correlate specific genera within fecal microbiota with plasma levels of pro-inflammatory cytokines and confirm the role of these cytokines in a large independent large cohort of patients with PD and controls. Our study also has some limitations. First, we did not check fecal short-chain fatty acid (SCFA) levels, which are a potential mediator of neurodegeneration caused by gut microbiome composition alterations [4], although the vast majority of studies suggest SCFAs play a beneficial and anti-inflammatory role in neurodegeneration [32]. A shotgun metagenome analysis could provide more detailed information about the microbiota, including the functional analysis, to analyze fecal microbiota in PD. Second, our study is a cross-sectional design, and we only considered diabetes mellitus and hypertension as major medical co-comorbidities in our study population. As many other comorbidities, for example, anxiety, depression, constipation, and other medical disorder, including rheumatoid arthritis and metabolic disorders, would also affect the gut microbiome, a longitudinal follow-up study considering all possible medical comorbidities and non-motor symptoms of PD will clarify the role of altered gut microbiota in disease progression. Finally, we did not analyze the correlations between every 
taxon and every cytokine listed in the panel due to the consideration of limited statistical power of multiple comparisons in the current study scale. We therefore only examined the taxa which was different between PD and controls to see the related changes of plasma cytokines under the hypothesis that altered microbiota may trigger systemic inflammatory responses contributing to PD. Future studies enrolling more participants and transcriptome assay to evaluate any possible changes of cytokines are needed to systemically explore the specific immune responses triggered by a particular genus or group of gut microorganisms.

\section{Conclusions}

We find that gut microbiota is altered in PD and correlate with clinical phenotypes and disease severity in our population. The altered plasma cytokine concentrations associated with specific changes in microbiota further demonstrate that altered microbiome composition alterations with aberrant host immune responses are linked with PD pathogenesis. Elucidating the interplay between microbial and host immune responses will lead to a better understanding of PD pathogenesis.

\section{Additional files}

Additional file 1 : Supplementary methods. (DOCX $21 \mathrm{~kb}$ )

Additional file 2 : Table S1 Clinical characteristics of the study participants. Table S2 General linear models for fecal genera based on differences between patients with PD and healthy controls. (DOCX 19 kb)

Additional file $\mathbf{3}$ : Figure S1 The plasma levels of individual cytokines in the second set of the study group. The plasma concentrations of IL-13, IFNy, and TNFa were significantly higher in PD patients compared to control participants in the second set of the study design. The error bar indicated standard error of mean (SEM). PD: Parkinson's disease. ${ }^{*} P<0.05$. **P $<0.01$. (JPG $127 \mathrm{~kb})$

\section{Abbreviations}

16S rRNA: 16S ribosomal RNA; ENS: Enteric nervous system; LB: Lewy bodies; LRRK2: Leucine-rich repeat kinase 2; MMSE: Mini-Mental State Examination; NMSS: Non-Motor Symptom assessment Scale; OTUs: Operational taxonomic units; PD: Parkinson's disease; PIGD: Postural instability and gait difficulty; UPDRS: Unified Parkinson's Disease Rating Scale

\section{Acknowledgements}

We thank all the subjects that participated in this study, the staff of the second core laboratory of National Taiwan University Hospital and the Medical Microbiota Center of the first core laboratory of National Taiwan University College of Medicine for their technical support.

\section{Authors' contributions}

$\mathrm{CHL}, \mathrm{CCC}$, and MSW were responsible for the study concept and design. $\mathrm{CHL}, \mathrm{CCC}, \mathrm{HLC}, \mathrm{JML}, \mathrm{CMC}, \mathrm{TPL}, \mathrm{EYC}, \mathrm{YCT}, \mathrm{CC}, \mathrm{HIL}$, and MSW acquired the data. $\mathrm{CHL}, \mathrm{CCC}, \mathrm{JML}, \mathrm{CMC}$, TPL, and EYC analyzed and interpreted the data. $\mathrm{CHL}$ and CCC drafted the manuscript. CHL critically revised the manuscript for important intellectual content and supervised the study. All authors read and approved the final manuscript.

\section{Funding}

We are grateful to the National Health Research Institutes (NHRI-EX10710716NC), the Taiwan Ministry of Science and Technology (MOST 106-2314-
B-002-227- and MOST 107-2320-B-002-032-), the National Taiwan University Hospital (106-EDN06 and 107-EDN14) and the Program for Translational Innovation of Biopharmaceutical Development-Technology Supporting Platform Axis (107-0210-01-19-04) for their funding support of this work.

\section{Availability of data and materials}

All data are available under request. The datasets used and analyzed during the current study are available from the corresponding author on reasonable request.

\section{Ethics approval and consent to participate}

All participants provided informed consent and the study was approved by the institutional ethics board.

\section{Consent for publication}

The paper has not been published previously and is not under consideration for publication elsewhere. All co-authors have read and approved the submission.

\section{Competing interests}

All authors declare that they have no competing interests.

\section{Author details}

${ }^{1}$ Department of Neurology, National Taiwan University Hospital, College of Medicine, National Taiwan University, Taipei 100, Taiwan. ${ }^{2}$ Department of Gastroenterology, National Taiwan University Hospital, College of Medicine, National Taiwan University, Taipei, Taiwan. ${ }^{3}$ Graduate Institute of Clinical Medicine, College of Medicine, National Taiwan University, Taipei, Taiwan. ${ }^{4}$ Department of Neurology, Taipei Veterans General Hospital, Taipei, Taiwan. ${ }^{5}$ Bioinformatics and Biostatistics Core, Center of Genomic and Precision Medicine, National Taiwan University, Taipei, Taiwan. ${ }^{6}$ Institute of Epidemiology and Preventive Medicine, National Taiwan University, Taipei, Taiwan. ${ }^{7}$ Graduate Institute of Biomedical Electronics and Bioinformatics, National Taiwan University, Taipei, Taiwan. ${ }^{8}$ Bioinformatics and Biostatistics Core, Center of Genomic Medicine, National Taiwan University, Taipei,

Taiwan. ${ }^{9}$ Department of Neurology, E-Da Hospital, Kaohsiung, Taiwan.

Received: 29 January 2019 Accepted: 20 June 2019

Published online: 27 June 2019

References

1. Kalia LV, Lang AE. Parkinson's disease. Lancet. 2015;386:896-912.

2. Braak H, Del Tredici K, Rüb U, de Vos RA, Jansen Steur EN, Braak E. Staging of brain pathology related to sporadic Parkinson's disease. Neurobiol Aging. 2003:24:197-211.

3. Abbott RD, Petrovitch H, White LR, Masaki KH, Tanner CM, Curb JD, et al. Frequency of bowel movements and the future risk of Parkinson's disease. Neurology. 2001;57:456-62.

4. Sampson TR, Debelius JW, Thron T, Janssen S, Shastri GG, Ilhan ZE, et al. Gut microbiota regulate motor deficits and Neuroinflammation in a model of Parkinson's disease. Cell. 2016;167:1469-80.

5. Unger MM, Spiegel J, Dillmann KU, Grundmann D, Philippeit H, Bürmann J, et al. Short chain fatty acids and gut microbiota differ between patients with Parkinson's disease and age-matched controls. Parkinsonism Relat Disord. 2016;32:66-72.

6. Klingelhoefer $\mathrm{L}$, Reichmann H. Pathogenesis of Parkinson disease-the gutbrain axis and environmental factors. Nat Rev Neurol. 2015;11:625-36.

7. Houser MC, Tansey MG. The gut-brain axis: is intestinal inflammation a silent driver of Parkinson's disease pathogenesis? NPJ Parkinsons Dis. 2017;3:3. https://doi.org/10.1038/s41531-016-0002-0.

8. Scheperjans F, Aho V, Pereira PA, Koskinen K, Paulin L, Pekkonen E, et al. Gut microbiota are related to Parkinson's disease and clinical phenotype. Mov Disord. 2015:30:350-8.

9. Keshavarzian A, Green SJ, Engen PA, Voigt RM, Naqib A, Forsyth CB, et al. Colonic bacterial composition in Parkinson's disease. Mov Disord. 2015;30: 1351-60.

10. Bedarf JR, Hildebrand F, Coelho LP, Sunagawa S, Bahram M, Goeser F, et al. Functional implications of microbial and viral gut metagenome changes in early stage L-DOPA-naive Parkinson's disease patients. Genome Med. 2017;9: 39. https://doi.org/10.1186/s13073-017-0428-y. 
11. Hopfner F, Künstner A, Müller SH, Künzel S, Zeuner KE, Margraf NG, et al. Gut microbiota in Parkinson disease in a northern German cohort. Brain Res. 2017;1667:41-5.

12. Scheperjans F. Gut microbiota, 1013 new pieces in the Parkinson's disease puzzle. Curr Opin Neurol. 2016;29:773-80.

13. Muangpaisan W, Hori H, Brayne C. Systematic review of the prevalence and incidence of Parkinson's disease in Asia. J Epidemiol. 2009;19:281-93.

14. Healy DG, Falchi M, O'Sullivan SS, Bonifati V, Durr A, Bressman S, et al. Phenotype, genotype, and worldwide genetic penetrance of LRRK2associated Parkinson's disease: a case-control study. Lancet Neurol. 2008;7: 583-90.

15. Paun A, Yau C, Danska JS. Immune recognition and response to the intestinal microbiome in type 1 diabetes. J Autoimmun. 2016;71:10-8.

16. Hughes AJ, Daniel SE, Kilford L, Lees AJ. Accuracy of clinical diagnosis of idiopathic Parkinson's disease: a clinico-pathological study of 100 cases. J Neurol Neurosurg Psychiatry. 1992;55:181-4.

17. Chiu TH, Huang HY, Chen KJ, Wu YR, Chiu JP, Li YH, et al. Relative validity and reproducibility of a quantitative FFQ for assessing nutrient intakes of vegetarians in Taiwan. Public Health Nutr. 2014;17:1459-66.

18. Longstreth GF, Thompson WG, Chey WD, Houghton LA, Mearin F, Spiller RC. Functional bowel disorders. Gastroenterology. 2006;130:1480-91.

19. Eren AM, Maignien L, Sul WJ, Murphy LG, Grim SL, Morrison HG, et al. Oligotyping: differentiating between closely related microbial taxa using $16 \mathrm{~S}$ rRNA gene data. Methods Ecol Evol. 2013;4(12). https://doi.org/10.1111/ 2041-210X.12114.

20. Ter Horst R, Jaeger M, Smeekens SP, Oosting M, Swertz MA, Li Y, et al. Host and environmental factors influencing individual human cytokine responses. Cell. 2016;167:1111-24.

21. Qian $Y$, Yang X, Xu S, Wu C, Song Y, Qin N, et al. Alteration of the fecal microbiota in Chinese patients with Parkinson's disease. Brain Behav Immun. 2018:70:194-202

22. Heintz-Buschart A, Pandey U, Wicke T, Sixel-Döring F, Janzen A, SittigWiegand $\mathrm{E}$, et al. The nasal and gut microbiome in Parkinson's disease and idiopathic rapid eye movement sleep behavior disorder. Mov Disord. 2018; 33:88-98.

23. Petrov VA, Saltykova IV, Zhukova IA, Alifirova VM, Zhukova NG, Dorofeeva YB, et al. Analysis of gut microbiota in patients with Parkinson's disease. Bull Exp Biol Med. 2017;162:734-7.

24. Hasegawa S, Goto S, Tsuji H, Okuno T, Asahara T, Nomoto K, et al. Intestinal dysbiosis and lowered serum lipopolysaccharide-binding protein in Parkinson's disease. PLoS One. 2015;10:e0142164.

25. Scher JU, Sczesnak A, Longman RS, Segata N, Ubeda C, Bielski C, et al. Expansion of intestinal Prevotella copri correlates with enhanced susceptibility to arthritis. Elife. 2013;2:e01202. https://doi.org/10.7554/eLife. 01202.

26. Lebouvier T, Neunlist M, Bruley d, Varannes S, Coron E, Drouard A, N'Guyen $J M$, et al. Colonic biopsies to assess the neuropathology of Parkinson's disease and its relationship with symptoms. PLoS One. 2010;5:e12728. https://doi.org/10.1371/journal.pone.0012728.

27. Choi VM, Herrou J, Hecht AL, Teoh WP. Turner JR, Crosson S, et al. Activation of Bacteroides fragilis toxin by a novel bacterial protease contributes to anaerobic sepsis in mice. Nat Med. 2016;22:563-7.

28. Engen PA, Dodiya HB, Naqib A, Forsyth CB, Green SJ, Voigt RM, et al. The potential role of gut-derived inflammation in multiple system atrophy. J Park Dis. 2017:7:331-46.

29. Delahooke DM, Barclay GR, Poxton IR. Tumor necrosis factor induction by an aqueous phenol-extracted lipopolysaccharide complex from Bacteroides species. Infect Immun. 1995;63:840-6.

30. Houser MC, Chang J, Factor SA, Molho ES, Zabetian CP. Hill-Burns EM, et al. Stool immune profiles evince gastrointestinal inflammation in Parkinson's disease. Mov Disord. 2018:33:793-804.

31. Wakil AE, Wang ZE, Ryan JC, Fowell DJ, Locksley RM. Interferon gamma derived from CD4(+) T cells is sufficient to mediate T helper cell type 1 development. J Exp Med. 1998;188:1651-6.

32. Tan AH, Chong CW, Song SL, Teh CSJ, Yap IKS, Loke MF, et al. Altered gut microbiome and metabolome in patients with multiple system atrophy. Mov Disord. 2018;33:174-6.

\section{Publisher's Note}

Springer Nature remains neutral with regard to jurisdictional claims in published maps and institutional affiliations.

\section{Ready to submit your research? Choose BMC and benefit from}

- fast, convenient online submission

- thorough peer review by experienced researchers in your field

- rapid publication on acceptance

- support for research data, including large and complex data types

- gold Open Access which fosters wider collaboration and increased citations

- maximum visibility for your research: over $100 \mathrm{M}$ website views per year

At BMC, research is always in progress.

Learn more biomedcentral.com/submissions 